\title{
Eco-genetic additivity of diploids in allopolyploid wild wheats
}

\author{
Stella Huynh ${ }^{1}$, Awaiting Activation ${ }^{2,3}$, Antoine Guisan ${ }^{3}$, Francois Felber ${ }^{4}$, and Christian \\ Parisod $^{1}$ \\ ${ }^{1}$ University of Bern \\ ${ }^{2}$ Institute of Earth Surface Dynamics (IDYST), University of Lausanne (UNIL) \\ ${ }^{3}$ University of Lausanne \\ ${ }^{4}$ Musée et Jardins botaniques cantonaux de Lausanne et Pont-de-Nant
}

April 28, 2020

\begin{abstract}
Underpinnings of the distribution of allopolyploid species (hybrids with duplicated genome) along spatial and ecological gradients are elusive. As allopolyploid speciation combines the range of genetic and ecological characteristics of divergent diploids, allopolyploids initially show their additivity and are predicted to evolve differentiated ecological niches to establish in face of their competition. Here, we use four diploid wild wheats that differentially combined into four independent allopolyploid species to test for such additivity and assess the impact of ecological constraints on species ranges. Divergent genetic variation from diploids being fixed in heterozygote allopolyploids supports their genetic additivity. Spatial integration of comparative phylogeography and modeling of climatic niches supports ecological additivity of locally adapted diploid progenitors into allopolyploid species which subsequently colonized wide ranges. Allopolyploids fill suitable range to a larger extent than diploids and conservative evolution following the combination of divergent species appears to support their expansion under environmental changes.
\end{abstract}

\section{Introduction}

Polyploidy is as an important mechanism of speciation and diversification in plants (Stebbins 1971; Wood et al. 2009). Allopolyploid species, derived from hybridization and whole genome duplication of diploids, are particularly widespread across both geographical and environmental spaces, but underpinnings of their large distribution ranges remain elusive (te Beest et al. 2012). Allopolyploids arise in sympatry with one or both of their progenitor species and nascent populations therefore have to struggle against a frequency-dependent disadvantage to reproduce and establish in face of locally adapted diploids (i.e. 'minority cytotype exclusion'; Levin 1975; Fowler \& Levin 1984). An influential prediction of this model is that successfully established polyploids have shifted their ecological niche to stably coexist with their diploid progenitors. Novel genetic and environmental traits exhibited by polyploids were accordingly often discussed as the result of such an adaptive path (Levin 2002; Soltis et al. 2016). Despite the hypothesis that non-equilibrium climates of the Quaternary have promoted polyploid speciation (Stebbins 1985), neutral models of allopolyploid evolution remain largely to be explored. To what extent increased availability of suitable space during climate-enforced range shifts may lessen the competition from diploid progenitors and thus support allopolyploid speciation without niche shift is unknown.

Several studies having contrasted geographical and ecological ranges of diploid vs polyploid species supported the climatic niche divergence predicted under competitive exclusion (e.g. Theodoridis et al. 2013; Ramsey \& Ramsey 2014). However, they rarely considered historical factors or tested specific hypotheses regarding the origin(s) of polyploids from multiple progenitor species. Despite recent work using ecological 
modeling across species to address the success of polyploids (e.g. Martin \& Husband 2009; Glennon et al. 2014; Blaine Marchant et al. 2016; Baniaga et al. 2019), the evolution of their ecological niches following the combination of diploids remains elusive. To account for the impact of hybridization between differentially adapted taxa, Parisod \& Broennimann (2016) suggested to not only rely on adequate phylogenies but to go beyond ambiguous pairwise comparisons by testing the null hypothesis that allopolyploids represent the additivity of their diploid progenitors. Allopolyploid speciation is indeed initiated by the combination of divergent suites of diploid loci that expectedly sum up as fixed heterozygosity in the derived allopolyploids (i.e. genetic additivity). Allopolyploid species correspondingly unite ecological tolerances from their progenitors and expectedly fully occupy their ecological niches (i.e. ecological additivity). Only significant deviation beyond such combined ranges of conditions is considered as characteristic of potential novelties supporting the establishment of allopolyploids in a distinct ecological niche.

This work uses wild wheats (Aegilops sp) to assess the eco-evolutionary drivers of range dynamics among four diploid species $(2 \mathrm{x}=14$ chromosomes) that differentially combined into four independent allopolyploid species $(4 \mathrm{x}=28$; Feldman \& Levy 2015). Selected species are all annual selfers that share a ruderal life history (van Slageren 1994). Diploids are however typically distributed across restricted ranges, whereas allopolyploid wild wheats are widespread throughout the Mediterranean area (Kilian et al. 2011). Here, we use this excellent model system to investigate the genetic and environmental additivity of diploid progenitors in allopolyploids and identify drivers of their successful establishment and large distribution ranges. Despite prior cytogenetic and phylogenetic studies (e.g. Meimberg et al. 2009; Bernhardt et al. 2017), diploid progenitors and the recurrent origins of allopolyploid wild wheats were not precisely identified. Accordingly, we first infer the reticulate phylogeny of allopolyploids using plastid and nuclear loci to provide a robust comparative framework. Nuclear loci among samples collected across native species ranges then address the genetic additivity of diploids in allopolyploids and compare their phylogeography to assess main processes having shaped genetic variation. Environmental modeling is finally used to compare climatic niches of species and test to what extent allopolyploids differ from the expected ecological additivity of their diploid progenitors. Our integration of genetic and environmental characteristics supports an overall additivity of locally adapted diploid progenitors in allopolyploid species that successfully expend during environmental changes.

\section{Material and Methods}

Sampling across distribution ranges of diploid and allopolyploid species

Four diploid species - Ae. caudata (genome CC), Ae. comosa (MM), Ae. tauschii (DD) and Ae. umbellulata (UU) - and four of their derived allopolyploid species - Ae. crassa (DDMM), Ae. cylindrica (DDCC), Ae. geniculata (UUMM) and Ae. triuncialis (UUCC) - were investigated. Selected species show clear morphological differences and are representative of the diversity of existing polyploid complexes in wild wheats (Kilian et al. 2011).

A total of 402 geo-localized accessions of the eight Aegilops spp. were selected from germplasms to represent genetic variation across distribution ranges with 30 and 80 accessions per diploid and polyploid species, respectively (Table S1). For Ae. crassa, only 10 accessions were available. DNA was extracted using

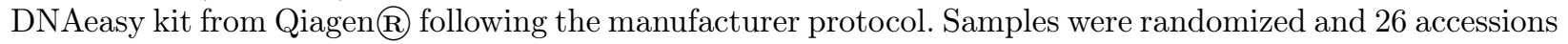
were replicated among 96-well plates to ensure genotyping accuracy. DNA concentration was measured by Nanodrop and normalized at $10 \mathrm{ng} / \mu \mathrm{L}$ and $50 \mathrm{ng} / \mu \mathrm{L}$ for amplification of plastid and nuclear loci, respectively.

Occurrences of the eight Aegilops species were collected from the GBIF database (http://www.gbif.org/) and filtered out from replicates and non-native samples from America (Lyons et al. 2010). Including genotyped samples, a total of 3667 occurrences georeferenced with a precision of $1 \mathrm{~km}$ (i.e. at least 4 decimal coordinates; Table S1) were kept for niche analyses. 
Genetic analyses of plastid and nuclear loci to infer origin(s) of allopolyploids

To address the reticulate evolutionary relationships among species and identify corresponding progenitor(s) of polyploid individuals, all accessions were sequenced at two maternally-inherited plastid loci and 30 biparentally-inherited nuclear loci (Petit et al. 2005; Li et al. 2019). Intergenic loci of the plastid genomes, $n d h F-r p l 32$ and $\operatorname{trn} T-\operatorname{trn} F$, were sequenced and concatenated following Meimberg et al. (2009) to infer the Median-Joining haplotype network shown in Fig S1 using SplitsTree4 (Huson \& Bryant 2006). Nuclear loci (Table S1) were genotyped by sequencing multiplexed amplicons encompassing non-coding introns of independent low-copy genes, using 48.48 Access Arrays from Fluidigm coupled with Illumina MiSeq (Text $\mathrm{S} 1)$. Allopolyploid samples were conservatively genotyped based on high-coverage sequences (2x250 bp) as $\mathrm{AAAA}, \mathrm{AABB}, \mathrm{ABC}$ - and $\mathrm{ABCD}$ for samples presenting one, two, three and four alleles, respectively.

To address the expected genetic additivity of progenitors in allopolyploids, nuclear haplotypes were assigned to their corresponding subgenomes and the relative contribution of each diploid species was estimated. At each locus, haplotypes segregating in polyploids that were identical to those in diploid progenitor species were considered as "conserved haplotypes" assigned to the corresponding subgenome or considered as shared when reported among diploids. Haplotypes specific to an allopolyploid were considered as "derived haplotypes" and assigned to the subgenome showing shorter branch lengths than with other diploids (Text S1). Derived haplotypes showing unclear phylogenetic relationships with putative progenitors were considered of unknown origin and lost, and coded as missing data. Under the null hypothesis that allopolyploids are the genetic additivity of progenitor species, haplotypes from each subgenome are expected to segregate in similar proportions. After normalization per loci, proportion tests assessed such predictions for conserved, derived and lost haplotypes segregating within the species.

Phylogenetic inferences and origins of allopolyploid species

To infer a reticulate species network, allopolyploid multi-labeled species trees were assessed from haplotype sequences at 30 nuclear loci (Text S1) using starBEAST in BEAST 2.5 (Drummond et al. 2012). The species trees were based on a subset of 39 representative samples selected as two random individuals from each genetic cluster within species (see Genetic structure) and two outgroups. Each allopolyploid subgenome was analyzed as a distinct diploid individual within the species and run up to 800 million generations of Markov Chain Monte Carlo (MCMC), until stable MCMC mixing after $10 \%$ burnin and tree sampling every 100,000 generations. Convergence was checked on Tracer v1.7 (Rambaut et al. 2018) and Maximum Credibility Clade trees were summarized by TreeAnnotator available in BEAST2 and viewed in FigTree v1.4.3 (http://tree.bio.ed.ac.uk/software/figtree/). A secondary calibration based on divergence of diploid Aegilops species following Huynh et al. (2019) was used to date each allopolyploid species tree, considering issues inherent to molecular dating of polyploidy events (Doyle \& Egan 2010).

Genetic structure within and among species

Allelic richness (AR) within species was estimated for both plastid and nuclear loci as the expected number of alleles among $\mathrm{k}$ gene copies (i.e. the minimum sample size of 10 and 40 gene copies reported in Ae. crassa for plastid and nuclear loci, respectively). Observed heterozygosity (Ho) was estimated as the average proportion of heterozygotes among nuclear loci within each species. To account for possible phylogenetic structure, gene diversity within species was estimated after correction for sample size following Nei (1978) from both unordered alleles ( $h$; i.e. all alleles considered equidistant) and ordered alleles ( $v$; i.e. considering phylogenetic distances as their proportion of nucleotide substitutions) using SPAGeDi (Hardy \& Vekemans 2002).

To highlight phylogeographic signals in each species, intraspecific genetic structure was assessed from nuclear 
loci using Bayesian clustering as implemented in STRUCTURE 2.3.4 (Pritchard et al. 2000). Based on the admixture model with correlated alleles, 500,000 MCMC generations with 100,000 burnin were repeated ten times for each number of genetic clusters $(\mathrm{K})$ between one and ten, and the optimal $\mathrm{K}$ was selected through ad hoc deltaK procedure estimating the rate of change in the log probability of data between successive $\mathrm{K}$ values (Evanno et al. 2005) as implemented in STRUCTURE HARVESTER (Earl \& vonHoldt 2012). The average individual assignment probabilities under the optimal K were estimated over 20 iterations of 1,000,000 MCMC generations and 100,000 burnin with the FullSearch algorithm in CLUMPP 1.1.2 (Jakobsson \& Rosenberg 2007) and represented by stacked bar plot generated in $\mathrm{R}$ using strataG v0.9.4 package (Archer et al. 2017).

\section{Climatic niches quantifications}

We compared the climatic niche of diploid and allopolyploid species in a simplified climatic space summarizing the gradients present in the 19 high resolution bioclimatic variables from the CHELSA database (30 arc sec, i.e. $1 \mathrm{~km}$ precision; Karger et al. 2017). On top of those 19 variables, similarly high resolution monthly 'soil water balance' variables from CGIAR database (Trabucco \& Zomer 2010) were averaged over months and included as a 20th variable (swc) in the analyses. In order to allow direct comparisons of niches among all species, we defined a study area as the adjacent biomes (WWF; Olson et al. 2001) where occurrences of Aegilops species were recorded. Large geographical areas without occurrences were further excluded (e.g. Russia, the desert biome in North Africa; Fig. S1). The resulting area was considered as the accessible area available to all species (sensu Barve et al. 2011) and corresponding values for all 20 bioclimatic variables were used to produce a common background for all species in subsequent climatic niche analyses (Broennimann et al. 2012). We then run a principal component analysis (PCA) of a random sample of 1,000,000 raster cells across the study area (i.e. representing the whole realized environment, see PCA-env in Broennimann et al. 2012) and used the two first PCA axes (PC1 and PC2) as climatic space.

Using the niche analysis functions of the R-package ecospat (Di Cola et al. 2017), the realized niche of each Aegilops species was modeled in a common gridded climatic space (each pixel of that gridded space representing a unique combination of climatic conditions) using Gaussian kernel density analyses that smooth the density of occurrences and produce niche models independent from the sampling effort or the geographic resolution of occurrences (Broennimann et al. 2012). We also quantified the niche of the pool of each pair of known diploid progenitors as the expected niche of allopolyploids. Niche centroid and breadth (i.e. dispersion) were estimated according to density of the modeled niche along principal components ( $\mathrm{PC} 1$ and $\mathrm{PC} 2)$.

To what extent species occupy all available sites with suitable conditions (range filling; Svenning and Skov 2004) was estimated as the ratio of the effectively occupied area to the suitable area for each species. Our occurrence dataset was not exhaustive (i.e. did not covered every pixel occupied by the species) and the occupied area was therefore estimated using a kernel density function with $5 \%$ threshold around occurrences in the R-package ks (Duong 2019). The suitable area of each species was calculated based on the projection of its climatic niche onto the geography across the study area (using the function 'ecospat.niche.zProjGeo' in ecospat) and delimited by discarding $10 \%$ of the gridded space with lowest occurrence densities. Range filling was estimated as the proportion of occupied out of suitable cells.

\section{Climatic niches comparisons}

Different metrics were used to compare inferred climatic niches. First, we compared their overlap using the D metric of Schoener (1968), which measures the similarity between niches of any species based on the density of occurrence modeled in each pixel of the background climatic space. The significance of niche overlaps D was tested through similarity tests following Warren et al. (2008), by randomly reallocating niche centroid of both species and recalculating D through 100 repetitions following Broennimann et al. (2012). The similarity test thus assessed whether the range of climatic conditions (i.e. climatic niche) occupied by 
one species was more similar to the niche occupied by the other species than would be expected by chance ( $a=0.05)$. Using the USE framework (niche unfilling, stability and expansion) of Guisan et al. (2014), we further quantified the proportion of the climate space that was used only by the first species, only by the second species and by both species.

Niche stability $\mathrm{S}$ is a metric of niche overlap that considers the presence/absence of species and not their occurrence density as with the D metric. Both the D and USE metrics are empirical approaches relying kernel density estimations on existing environments and which calculate non-parametric niche shapes. These two metrics are thus robust to possibly non-convex niches and expectedly outperform alternative methods assuming niche convexity such as analyses of variance (Treier et al. 2009) or range bagging (Drake 2015). However, we further assessed niche differences among species based on 1000 bootstrap of niche centroids and breadths along PC1 and PC2 and comparisons using one-way ANOVA and post-hoc Tukey tests in the R-package vegan (Oksanen et al. 2019). Each bioclimatic factor was similarly compared among species.

\section{Additivity of allopolyploid climatic niches}

The null hypothesis of allopolyploid niche additivity (i.e. the combination of diploid progenitors' niches, thereafter the expected allopolyploid niche) was tested by comparing the observed allopolyploid niche to the expected niche modeled from combined occurrences of both its progenitors. The two metrics $\mathrm{D}$ and $\mathrm{S}$ of niche overlaps between the observed and expected niches of the allopolyploid species were estimated as before, with niche similarity test for D. Similarly, observed and expected niche centroids and breadths were compared using ANOVA and Tukey tests.

For each allopolyploid species, differences between the observed and the expected climatic niches were decomposed following the USE framework, and niche stability S thus corresponds to the fraction of the observed allopolyploid niche overlapping with the combination of both progenitors' niches. The fraction of the observed allopolyploid niche deviating from the additivity of progenitors (i.e. niche shift) was decomposed into niche contraction $U$ (i.e. proportion of unoccupied space as compared to expectations) and niche expansion E (i.e. proportion of newly occupied space).

Isolation-by-distance and isolation-by-environment in diploid and allopolyploid species

To what extent demographic vs ecological processes shaped genetic variation was addressed by signals of genetic isolation-by-distancevs isolation-by-environment within each species (Wang et al. 2013). Patterns of isolation-by-distance expected under restricted dispersal of neutral genes were tested by comparing Euclidian geographic and genetic pairwise distances through Mantel tests with 20,000 permutations in vegan . Genetic distances among individuals were estimated using Rousset's a statistics (analogous to FST/(1-FST)) in SPAGeDi (Hardy \& Vekemans 2002).

Patterns of isolation-by-environment expected when selection favors gene exchanges among individuals from similar climatic conditions (Wanget al. 2013; Wang \& Bradburd 2014) were tested through partial Mantel tests associating genetic, geographic and climatic distances invegan. Following Legendre \& Fortin (2010), correlations of genetic distances with Euclidian distances from PCs of the common climatic space while accounting for joint spatial structure were interpreted as isolation-by-environment. Provided the questioned performance of such tests (e.g. Legendre et al . 2015), corresponding patterns were confirmed through multivariate Redundancy Analysis (RDA) associating individual allelic frequencies to the geographical coordinates and/or coordinates along PCs of the climatic space with 20,000 permutations in the R-package adespatial (Drayet al. 2019).

\section{Results}

Species phylogeny and genetic additivity of diploids in allopolyploids

Amplicon sequencing of 402 accessions from the eight diploid-allopolyploid Aegilops species yielded robust 
allelic variation at plastid loci (Fig. S2) and at 30 low-copy nuclear genes (Text S1, Fig. S3). Phylogenies of plastid and nuclear loci among representative samples resolved species in monophyletic clades and unambiguously related the different allopolyploids to their respective maternal $\mathrm{x}$ paternal diploid progenitors (Fig. 2A). Consistent with taxonomy, multi-labeled nuclear species trees accounting for reticulation related Ae. crassa (DDMM) to ancestors of Ae. comosa (MM) and other diploids that have hybridized with Ae. tauschii (DD). Divergent nuclear haplotypes within Ae. crassa $\left(\mathrm{Cr}_{2} \mathrm{k} 2\right)$ were related to distinct diploid lineages and included hexaploids (DDDDMM) of more recent origin (Fig. S3 and S4). Phylogenies further supported independent origins of multiple lineages within all allopolyploid species. Nuclear loci indicated an origin of Ae. geniculata (UUMM) combining ancestors of Ae. umbellulata (UU; identified as the maternal progenitor by plastid loci) and of Ae. comosa (MM), with specific samples presenting divergent sequences from distinct diploid lineages and supporting multiple origins of this allopolyploid. Multiple origins of Ae. triuncialis (UUCC) were patent from plastid haplotypes shared with both diploids Ae. caudata (CC) and Ae. umbellulata (UU) and nuclear loci congruently supporting such bidirectional hybridization at the origin of this allopolyploid (Fig. S5). Independent maternal lineages of Ae. cylindrica (DDCC) were supported by distinct plastid haplotypes of Ae. tauschii (DD) and nuclear loci that identified Ae. caudata (CC) as the paternal progenitor. Allopolyploids originated at different times, as indicated by the divergence of their alleles from corresponding diploids (Fig. S3). Cautious interpretations of molecular dating show that allopolyploids Ae. crassa andAe. geniculata originated more than 1 Mya, whereas youngerAe. cylindrica and Ae. triuncialis appeared later $(>0.2 \mathrm{Mya})$ and also went through repeated range contraction-expansion during the late Pleistocene.

Based on this phylogenetic framework, allopolyploid species (exceptAe. crassa whose ancestral maternal progenitor is elusive) were consistent with the expected genetic additivity of their diploid progenitors and presented similar proportions of segregating haplotypes from each subgenome (Table S2). Patterns of genetic additivity were further consistent with the relative age of allopolyploid species, with oldest species such as Ae. geniculata showing $41.9 \%$ of haplotypes from both subgenomes having diverged from diploids, whereas youngest species such as Ae. cylindrica presented only $3.6 \%$ of derived and mostly (94.3\%) conserved haplotypes (Fig. 2B).

Genetic structure within diploid and allopolyploid species

Variation segregating at nuclear loci within species was congruent with the hypothesis of genetic additivity. Proxies of genetic diversity were consistently higher in allopolyploids $(\mathrm{AR}=2.3-5.9, \mathrm{~h}=0.50-0.63$ and $v=$ $0.13-0.017)$ than in their respective progenitor diploids $(\mathrm{AR}=2.78-4.77, \mathrm{~h}=0.33-0.50$ and $v=0.002-0.007$; Table 1). Allopolyploid species showed not only higher phylogenetic diversity $(v)$ than diploids but also the pattern of fixed heterozygosity $(\mathrm{Ho}=0.56-0.66)$ expected by the strict combination of divergent diploid loci.

To address phylogeographic processes having shaped genetic variation in diploids and allopolyploids, the genetic structure across the distribution range of each species was inferred using STRUCTURE (Fig. 3). Most species presented an optimal number of two or three genetic clusters and high genetic diversity across Anatolia (Fig. S4). Multiple spatially-coherent genetic clusters were co-existing within all species across that region, whereas genetically admixed individuals indicative of secondary contact between intraspecific lineages were mostly reported in the Thyracian plain (between the Balkans and Western Anatolia), the Taurus chain (between Western and Eastern Anatolia) and the Anatolian Diagonal (separating Eastern Anatolia from the Irano-Turanian region). In contrast to expectation based on their relative age, all diploid species presented locally differentiated genetic clusters across their restricted range, whereas polyploid species showed large areas outside Anatolia dominated by specific genetic clusters. Such phylogeographic patterns are consistent with cycles of range contraction-expansion having shaped coherent hotspots and melting spots of genetic diversity among wild wheats.

Climatic niches of diploids and allopolyploids

The climatic niche of each Aegilops species was modeled in a common background based on 20 bioclimatic factors and that summarized $70.69 \%$ of the variation along its first two principal components representative 
of the Mediterranean-temperate vs.Atlantic-continental climatic gradients (Fig. 4).

Comparative niche modeling addressed the hypothesis that allopolyploids are the ecological additivity of diploid species. Climatic niches modeled among the eight species showed specific but consistent D overlap (Fig. S6 and Table S3) and niche stability S values ranging from 0.39 to 0.93 (Fig. 4). Diploid species presented divergent climatic niche centroids, with Ae. tauschii and Ae. umbellulata being more continental than $A$ e. caudata and Ae. comosa, and relatively low niche breadths (Table S4). In contrast, all allopolyploid species, except Ae. crassa, presented relatively broad climatic niches that were largely coherent with niche additivity of their progenitors (Fig. 4). The observed niche of allopolyploids presented higher similarity to the one predicted based on diploids than expected by chance (Table S3). Alternative insights from bootstraping of observed niche centroids confirmed this pattern with significant but generally low differences to the expected niche centroids based on progenitors (Table S4; Fig. S7). Similarly, independent bioclimatic factors rarely supported different preferences in allopolyploids as compared to diploids (Table S4). Alternative approaches were thus largely congruent and indicate that allopolyploids occupy a combination of climatic ranges from their diploid progenitors.

Decomposition of the climatic niche of allopolyploids as compared to the combination of diploid ones confirmed niche conservatism in allopolyploid wild wheats (Fig. 4). They indeed presented substantial niche stability $(\mathrm{S}=0.72-0.93)$ and limited evidence of niche contraction ( $\mathrm{U}=0.01-0.18)$. Evidence of niche expansion $(\mathrm{E}=0.06-0.19)$ indicated that some allopolyploids occupied conditions beyond the combined range of diploid progenitors toward novel temperate or continental climates. Corresponding areas were however mostly located near range margins and were rarely occupied over significant geographic space (Fig. S8), contrasting with predictions that allopolyploids fill a new ecological niche. Only Ae. geniculata showed noticeable niche expansion across aridity gradients of Palestine, suggesting colonization of novel environments.

The geographical projection of modeled niches was coherent with the distribution ranges of each species and, as expected under non-equilibrium, suitable sites were not all occupied (Fig. S9). Large areas with suitable conditions were available for all species in the Iberian Peninsula or North Africa, although only allopolyploids densely occupy such regions. Range filling further assessed how the different species were distributed across space with tolerable conditions and showed that diploids effectively occupied only a low fraction of their suitable areas (0.05 to 0.25$)$ as compared to allopolyploids ( 0.40 and 0.51 ; Table 1$)$. Being robust to variable sampling effort (Fig. S10), such estimates indicate a greater filling of suitable space by allopolyploids than diploids.

\section{Isolation-by-distance and isolation-by-environment}

Associating genetic variation with spatial and ecological gradients, we assessed to what extent phylogeographic patterns of diploid and polyploid species were driven by neutral vs adaptive processes. Consistent with genetic variation shaped by neutral gene flow among individuals, both Mantel tests and RDA consistently showed significant pattern of isolation-by-distance within all diploid species, exceptAe. tauschii (Table 1). All allopolyploid species, exceptAe. cylindrica, contrastingly presented non-significant (Mantel test) or weak (RDA) patterns of isolation by distance, as expected when closely-related individuals expanded across wide geographical space.

Taking the spatial component of both genetic and climatic variation into account with partial Mantel tests and RDA, significant patterns of isolation-by-environment were observed within all diploid species. Allopolyploids, except Ae. triuncialis, contrastingly presented non-significant isolation-by-environment, suggesting a limited impact of climatic drivers in shaping genetic variation in such species. Overall, the distribution of genetic variation within species is consistent with locally adapted diploid progenitors and allopolyploid species having successfully expanded across large distribution ranges during environmental changes. 


\section{Discussion}

Comparative phylogeography of diploid and allopolyploid wild wheats

Hybridization of diploid wild wheat species produced differential combinations of divergent genomes in allopolyploid species, offering a fruitful comparative system. Plastid and nuclear loci from across species ranges generally offered insights coherent with taxonomy (reviewed in Feldman \& Levy 2015) and highlighted multiple origins of allopolyploid species through recurrent hybridization with the same maternal progenitor species (Ae. geniculata, Ae. cylindrica) or bidirectional hybridization with both progenitor species $($ Ae. triuncialis ). Phylogenetic insights further supported origins of allopolyploid wild wheats at different times during the late Quaternary, with Ae. crassa and Ae. geniculata being older than Ae. triuncialis and Ae. cylindrica. A conservative interpretation of molecular dating (Doyle \& Egan 2010) is that all diploid species and their allopolyploid derivatives went through multiple cycles of range expansion-contraction following Pleistocene climatic oscillations (Hewitt 2000).

Historical changes in distribution ranges have shaped genetic variation within both diploids and allopolyploids, and promoted long-term maintenance of phylogenetic diversity across the Anatolian region connecting the Mediterranean and the Irano-Turanian flora (Zohary 1973; Hegazy \& Lovett Doust 2016). Diploid wild wheats are restricted to areas where populations likely survived the ice age (Stewart et al. 2010) and, there, show locally differentiated genetic clusters. As allopolyploids also present most of their genetic clusters there, Anatolia appears as an evolutionary cradle having fostered polyploid speciation (Mohammadin et al . 2017). However, in contrast to diploids, allopolyploid species have expended far beyond Anatolia with genetically homogeneous lineages currently occupying large areas towards the west and/or the east. Such a pattern typically left by range expansion (Excoffier et al. 2009) indicates that allopolyploids have efficiently colonized new territories during the late Pleistocene. Only the ancient Ae. crassa seemingly declined throughout its prior range despite additional rounds of polyploidy and represents an exception among otherwise successful allopolyploid species having established across the whole Mediterranean area. Such comparative phylogeography supports a critical impact of historical demography in shaping variation in diploid and allopolyploid wild wheats under climate changes.

Impact of climatic constraints on the distribution of diploids and allopolyploids

According to neutral expectations, allopolyploid wild wheats combined genetic variation from both their diploid progenitors as fixed heterozygosity across loci. When accounting for existing environments matching the combined tolerances of diploids (i.e. non-convex nichesensu Drake 2015), allopolyploids filled climatic conditions more similar to the combined ranges of their progenitors than could be expected by chance. Although other environmental factors such as biotic interactions may underlie niche novelties not captured here (Wiszet al. 2013; Anderson 2017), the observed pattern supports conservative niche evolution in allopolyploids and contrasts with the significant niche differentiation predicted under their competitive exclusion by diploids (Fowler \& Levin 1984).

Diploid wild wheats typically presented patterns of genetic isolation-by-environment suggestive of divergent gene pools adapted to local climatic conditions (Funk et al. 2011), whereas allopolyploids presented weak spatial or environmental patterns rather coherent with their expansion over large areas (Castric \& Bernatchez 2003). More generally, divergent evolution of climatic tolerance in allopolyploids was supported here by only few occurrences showing niche novelties at range margins and rarely coincided with noticeable expansion. Only Ae. geniculata significantly spread into locally semi-arid environments of Palestine, where local hybridization withAe. triuncialis (Senerchia et al. 2016) may have promoted adaptive introgression, as shown in other species (Arnold et al.2016). In contrast to repetitive elements showing large genomic changes in allopolyploid wheats (e.g. Levy \& Feldman 2004; Senerchia et al. 2014), the here used low-copy loci emphasized long-term neutral divergence and thus established ecotypes. While our data ideally link genetic and environmental changes at a biogeographic scale, further studies should address the interplay between spatial expansion and adaptive radiation at finer scales (Pannell \& Fields 2014). 
Drivers of allopolyploid expansion

Following range dynamics of the late Pleistocene, allopolyploid wild wheats have spread beyond their sites of origin and, despite their younger age, currently occupy wider geographical space than their diploid progenitors. Genetic and ecological patterns congruently emphasize processes having conserved the additivity of progenitors. The combination of genetic variation from diploid species being differentially adapted to climatic conditions accordingly supported the expansion of allopolyploids (except the contracting Ae. crassa ) across a large range of conditions. Unlike novelties lessening competition from diploid progenitors, conservative evolution of allopolyploids was seemingly favored under historical demographic fluctuations and here supported consistent occupancy of suitable space, whereas diploid species remained restricted to a small fraction of their suitable range.

Being selfing ruderals with diaspores promoting dispersal, all wild wheat species were offered similar opportunities to expand with climate changes and the rise of agriculture (Salamini et al. 2002). The two times higher filling of potential range exhibited by allopolyploids is therefore consistent with an intrinsic advantage conferred by such a genetic system. Fixed heterozygosity in allopolyploids is likely key as it masks recessive alleles and thus reduces expression of the genetic load to support successful expansion of populations through recurrent founder events (Soltis \& Soltis 2000; Brochmann et al. 2004). Successful invasion of new territories by allopolyploid species such as Centaurea stoebe (Treier et al. 2009; Mráz et al. 2012) has accordingly been associated with reduced inbreeding depression (Rosche et al. 2017). Wild wheats here suggest that allopolyploidy supports climate-enforced range shifts and calls for additional knowledge on inbreeding depression across distribution ranges of diploid-polyploid systems to understand drivers of their range dynamics. More generally, to what extent niche divergence commonly described in allopolyploids deviates from the here supported genetic and ecological conservatism and may significantly reduce competition from progenitors remains largely unknown. Furthermore, autopolyploids may similarly benefit from increased heterozygosity under climate changes (Parisod et al. 2010), although studies in Chamerion angustifolium rather indicated adaptive evolution of eco-physiological traits and niche divergence (Maherali et al. 2009; Thompson et al. 2014). It is not our intention to here offer comparisons along the continuum of polyploid systems or to explore macro-evolutionary patterns, although we notice that the developed framework can promote generalization with larger sets of species (e.g. Baniaga et al . 2019; Burton et al. 2010; Rice et al. 2019). Fostering integration of historical and ecological constraints, it will shed light on the possible advantages of species resulting from pervasive polyploidy.

\section{Acknowledgments}

This work was funded by the Swiss National Science Foundation (31003A-153388). Data analyzed in this paper were generated at the Genetic Diversity Centre, ETH Zurich. We thank J.-C. Walser and T. Marcussen for their help with this multilocus dataset as well as E. Allan, S. Grünig and three anonymous reviewers for their valuable comments on the manuscript.

\section{References}

Anderson, R.P. (2017). When and how should biotic interactions be considered in models of species niches and distributions? J. Biogeogr. , 44, 8-17.

Archer, F.I., Adams, P.E. \& Schneiders, B.B. (2017). strataG: An r package for manipulating, summarizing and analysing population genetic data. Mol. Ecol. Resour., 17, 5-11.

Arnold, B.J., Lahner, B., DaCosta, J.M., Weisman, C.M., Hollister, J.D., Salt, D.E., et al. (2016). Borrowed alleles and convergence in serpentine adaptation. Proc. Natl. Acad. Sci. U. S. A., 113, 8320-8325. 
Baniaga, A.E., Marx, H.E., Arrigo, N. \& Barker, M.S. (2019). Polyploid plants have faster rates of multivariate niche differentiation than their diploid relatives. Ecol. Lett., https://doi.org/10.1111/ele.13402.

Barve, N., Barve, V., Jiménez-Valverde, A., Lira-Noriega, A., Maher, S.P., Peterson, A.T., et al. (2011). The crucial role of the accessible area in ecological niche modeling and species distribution modeling. Ecol. Model. , 222, 1810-1819.

te Beest, M., Le Roux, J.J., Richardson, D.M., Brysting, A.K., Suda, J., Kubesova, M., et al. (2012). The more the better? The role of polyploidy in facilitating plant invasions. Ann. Bot., 109, 19-45.

Bernhardt, N., Brassac, J., Kilian, B. \& Blattner, F.R. (2017). Dated tribe-wide whole chloroplast genome phylogeny indicates recurrent hybridizations within Triticeae. BMC Evol. Biol. , 17, 141.

Blaine Marchant, D., Soltis, D.E. \& Soltis, P.S. (2016). Patterns of abiotic niche shifts in allopolyploids relative to their progenitors. New Phytol. , 212, 708-718.

Brochmann, C., Brysting, A.K., Alsos, I.G., Borgen, L., Grundt, H.H., Scheen, A.-C., et al. (2004). Polyploidy in arctic plants.Biol. J. Linn. Soc. , 82, 521-536.

Broennimann, O., Fitzpatrick, M.C., Pearman, P.B., Petitpierre, B., Pellissier, L., Yoccoz, N.G., et al. (2012). Measuring ecological niche overlap from occurrence and spatial environmental data.Glob. Ecol. Biogeogr. , 21, 481-497.

Burton, O.J., Phillips, B.L. \& Travis, J.M.J. (2010). Trade-offs and the evolution of life-histories during range expansion. Ecol. Lett. , 13, 1210-1220.

Castric, V. \& Bernatchez, L. (2003). The rise and fall of isolation by distance in the anadromous brook charr (Salvelinus fontinalis Mitchill). Genetics , 163, 983-996.

Di Cola, V., Broennimann, O., Petitpierre, B., Breiner, F.T., D'Amen, M., Randin, C., et al. (2017). ecospat: an $\mathrm{R}$ package to support spatial analyses and modeling of species niches and distributions.Ecography , 40, $774-787$.

Doyle, J.J. \& Egan, A.N. (2010). Dating the origins of polyploidy events. New Phytol. , 186, 73-85.

Drake, J.M. (2015). Range bagging: a new method for ecological niche modelling from presence-only data. J. Roy. Soc. Interface, 12, 20150086.

Dray, S., Bauman, D., Blanchet, G., Borcard, D., Clappe, S. \& Guenard, G. (2019). adespatial: Multivariate Multiscale Spatial Analysis. $R$ package version 0.3-7. https://CRAN.R-project.org/package=adespatial. R.

Drummond, A.J., Suchard, M.A., Xie, D. \& Rambaut, A. (2012). Bayesian phylogenetics with BEAUti and the BEAST 1.7. Mol. Biol. Evol. , 29, 1969-1973.

Duong, T. (2019). ks: Kernel Smoothing. R package version 1.11.4.https:/CRAN.R-project.org/package=ks.

Earl, D.A. \& vonHoldt, B.M. (2012). STRUCTURE HARVESTER: a website and program for visualizing STRUCTURE output and implementing the Evanno method. Conserv. Genet. Resour. , 4, 359-361.

Evanno, G., Regnaut, S. \& Goudet, J. (2005). Detecting the number of clusters of individuals using the software structure: a simulation study. Mol. Ecol. , 14, 2611-2620.

Excoffier, L., Foll, M. \& Petit, R.J. (2009). Genetic consequences of range expansions. Annu. Rev. Ecol. Evol. Syst. , 40, 481-501.

Feldman, M. \& Levy, A.A. (2015). Origin and evolution of wheat and related Triticeae species. In: Alien introgression in wheat (eds. Molnár-Láng, M., Ceoloni, C. \& Doležel, J.). Springer, Cham, pp. 21-76.

Fowler, N.L. \& Levin, D.A. (1984). Ecological constraints on the establishment of a novel polyploid in competition with its diploid progenitor. Am. Nat. , 124, 703-711. 
Funk, D.J., Egan, S.P. \& Nosil, P. (2011). Isolation by adaptation inNeochlamisus leaf beetles: host-related selection promotes neutral genomic divergence. Mol. Ecol. , 20, 4671-4682.

Glennon, K.L., Ritchie, M.E. \& Segraves, K.A. (2014). Evidence for shared broad-scale climatic niches of diploid and polyploid plants.Ecol. Lett. , 17, 574-582.

Guisan, A., Petitpierre, B., Broennimann, O., Daehler, C. \& Kueffer, C. (2014) Unifying niche shift studies: insights from biological invasions. Trends Ecol. Evol. , 29, 260-269.

Hardy, O.J. \& Vekemans, X. (2002). SPAGeDi: a versatile computer program to analyse spatial genetic structure at the individual or population levels. Mol. Ecol. Notes , 2, 618-620.

Hegazy, A.K. \& Lovett Doust, J. (2016). Plant ecology in the Middle East . Oxford University Press, Oxford, United Kingdom.

Hewitt G. (2000). The genetic legacy of the Quaternary ice ages.Nature , 405, 907-913.

Huson, D.H. \& Bryant, D. (2006). Application of phylogenetic networks in evolutionary studies. Mol. Biol. Evol. , 23, 254-267.

Huynh, S., Marcussen, T., Felber, F. \& Parisod, C. (2019). Hybridization preceded radiation in diploid wheats. Mol. Phyl. Evol. , 139, 106554.

Jakobsson, M. \& Rosenberg, N.A. (2007). CLUMPP: a cluster matching and permutation program for dealing with label switching and multimodality in analysis of population structure. Bioinformatics , 23, 1801-1806.

Karger, D.N., Conrad, O., Böhner, J., Kawohl, T., Kreft, H., Soria-Auza, R.W., et al. (2017). Climatologies at high resolution for the earth's land surface areas. Sci. Data , 4, 170122.

Kilian, B., Mammen, K., Millet, E., Sharma, R., Graner, A., Salamini, F., et al. (2011). Aegilops. In: Wild Crop Relatives: Genomic and Breeding Resources (ed. Kole, C.). Springer Berlin, Heidelberg, pp. 1-76.

Legendre, P. \& Fortin, M.-J. (2010). Comparison of the Mantel test and alternative approaches for detecting complex multivariate relationships in the spatial analysis of genetic data. Mol. Ecol. Resour., 10, 831-844.

Legendre, P., Fortin, M.-J. \& Borcard, D. (2015). Should the Mantel test be used in spatial analysis? Meth. Ecol. Evol. , 6, 1239-1247.

Levin, D.A. (1975). Minority cytotype exclusion in local plant populations. Taxon, 24, 35-43.

Levin, D.A. (2002). The role of chromosomal change in plant evolution . Oxford University Press, New York.

Levy, A.A. \& Feldman, M. (2004). Genetic and epigenetic reprogramming of the wheat genome upon allopolyploidization. Biol. J. Linn. Soc., 82, 607-613.

Li, C., Sun, X., Conover, J.L., Zhang, Z., Wang, J., Wang, X., et al. (2019). Cytonuclear coevolution following homoploid hybrid speciation in Aegilops tauschii . Mol. Biol. Evol. , 36, 341-349.

Lyons, K.G., Shapiro, A.M. \& Schwartz, M.W. (2010). Distribution and ecotypic variation of the invasive annual barb goatgrass (Aegilops triuncialis ) on serpentine soil. Invasive Plant Sci. Manag. , 3, 376-389.

Maherali, H., Walden, A. E. \& Husband, B. C. (2009). Genome duplication and the evolution of physiological responses to water stress. New Phytol ., 184, 721-731.

Martin, S. L., \& Husband, B. C. (2009). Influence of phylogeny and ploidy on species ranges of North American angiosperms. J. Ecol. , 97, 913-922.

Meimberg, H., Rice, K.J., Milan, N.F., Njoku, C.C. \& McKay, J.K. (2009). Multiple origins promote the ecological amplitude of allopolyploid Aegilops (Poaceae). Am. J. Bot. , 96, 1262-1273. 
Middleton, C.P., Senerchia, N., Stein, N., Akhunov, E.D., Keller, B., Wicker, T., et al. (2014). Sequencing of chloroplast genomes from wheat, barley, rye and their relatives provides a detailed insight into the evolution of the Triticeae tribe. PLoS ONE, 9, e85761.

Mohammadin, S., Peterse, K., van de Kerke, S. J., Chatrou, L. W., Dönmez, A. A., Mummenhoff, K., et al. (2017). Anatolian origins and diversification of Aethionema, the sister lineage of the core Brassicaceae. Am. J. Bot. , 104, 1042-1054.

Mráz, P., Garcia-Jacas, N., Gex-Fabry, E., Susanna, A., Barres, L. \& Müller-Schärer, H. (2012). Allopolyploid origin of highly invasiveCentaurea stoebe s.l. (Asteraceae). Mol. Phylogenet. Evol. , 62, 612-623.

Nei, M. (1978). Estimation of average heterozygosity and genetic distance from a small number of individuals. Genetics , 89, 583-590.

Oksanen, J., Blanchet, F.G., Friendly, M., Kindt, R., Legendre, P., McGlinn, D., et al. (2019). vegan: Community Ecology Package. $R$ package version 2.4-4.https://CRAN.R-project.org/package=vegan.

Olson, D.M., Dinerstein, E., Wikramanayake, E.D., Burgess, N.D., Powell, G.V.N., Underwood, E.C., et al. (2001). Terrestrial ecoregions of the world: a new map of life on Earth. BioScience, 51, 933-938.

Pannell, J.R. \& Fields, P.D. (2014). Evolution in subdivided plant populations: concepts, recent advances and future directions. New Phytol. , 201, 417-432.

Parisod, C. \& Broennimann, O. (2016). Towards unified hypotheses of the impact of polyploidy on ecological niches. New Phytol. , 212, 540-542.

Parisod, C., Holderegger, R. \& Brochmann, C. (2010). Evolutionary consequences of autopolyploidy: Research review. New Phytol. , 186, 5-17.

Petit, R.J., Duminil, J., Fineschi, S., Hampe, A., Salvini, D., \& Vendramin, G. G. (2005). Comparative organization of chloroplast, mitochondrial and nuclear diversity in plant populations. Mol. Ecol. , 14, 689701.

Pritchard, J.K., Stephens, M. \& Donnelly, P. (2000). Inference of population structure using multilocus genotype data. Genetics , 155, 945-959.

Rambaut, A., Drummond, A.J., Xie, D., Baele, G. \& Suchard, M.A. (2018). Posterior summarization in Bayesian phylogenetics using Tracer 1.7.Syst. Biol. , 67, 901-904.

Ramsey, J. \& Ramsey, T.S. (2014). Ecological studies of polyploidy in the 100 years following its discovery. Philos. Trans. R. Soc. B Biol. Sci., 369, 20130352-20130352.

Rice, A., Šmarda, P., Novosolov, M., Drori, M., Glick, L., Sabath, N., et al. (2019). The global biogeography of polyploid plants. Nat. Ecol. Evol. , 3, 265-273.

Rosche, C., Hensen, I., Mráz, P., Durka, W., Hartmann, M., \& Lachmuth, S. (2017). Invasion success in polyploids: the role of inbreeding in the contrasting colonization abilities of diploid versus tetraploid populations of Centaurea stoebe s.l. J. Ecol. , 105, 425-435.

Salamini, F., Ozkan, H., Brandolini, A., Schäfer-Pregl, R. Martin, W. (2002). Genetics and geography of wild cereal domestication in the near east. Nat. Rev. Genet. , 3, 429-441.

Schoener, T.W. (1968). The Anolis lizards of Bimini: resource partitioning in a complex fauna. Ecology , 49, 704-726.

Senerchia, N., Felber, F., North, B., Sarr, A., Guadagnuolo, R. \& Parisod, C. (2016). Differential introgression and reorganization of retrotransposons in hybrid zones between wild wheats. Mol. Ecol. , 25, 2518-2528.

Senerchia, N., Felber, F. \& Parisod, C. (2014). Contrasting evolutionary trajectories of multiple retrotransposons following independent allopolyploidy in wild wheats. New Phytol. , 202, 975-985. 
van Slageren, M.W.S.J.M. (1994). Wild wheats: a monograph of Aegilops L. and Amblyopyrum (Jaub. $\mathscr{B}$ Spach) Eig (Poaceae): a revision of all taxa closely related to wheat, excluding wild Triticum species, with notes on other genera in the tribe Triticcae, especially Triticum. Wageningen Agricultural University papers, Wageningen.

Soltis, D.E., Visger, C.J., Marchant, D.B. \& Soltis, P.S. (2016). Polyploidy: pitfalls and paths to a paradigm. Am. J. Bot. , 103, 1146-1166.

Soltis, P.S. \& Soltis, D.E. (2000). The role of genetic and genomic attributes in the success of polyploids. Proc. Natl. Acad. Sci. U. S. A., 97, 7051-7057.

Stebbins, G. L. (1985). Polyploidy, hybridization, and the invasion of new habitats. Ann. Miss. Bot. Gard. , 72, 824-832.

Stebbins, G.L. (1971). Chromosomal evolution in higher plants . University Park Press, London.

Stewart, J.R., Lister, A.M., Barnes, I. \& Dalén, L. (2010). Refugia revisited: individualistic responses of species in space and time.Proc. R. Soc. B Biol. Sci. , 277, 661-671.

Svenning, J. \& Skov, F. (2004). Limited filling of the potential range in European tree species. Ecology Letters $, 7,565-573$.

Theodoridis, S., Randin, C., Broennimann, O., Patsiou, T. \& Conti, E. (2013). Divergent and narrower climatic niches characterize polyploid species of European primroses in Primula sect. Aleuritia .J. Biogeogr. , 40, 1278-1289.

Thompson, K. A., Husband, B. C. \& Maherali, H. (2014). Climatic niche differences between diploid and tetraploid cytotypes of Chamerion angustifolium (Onagraceae). Am. J. Bot., 101, 1868-1875.

Trabucco, A. \& Zomer, R.J. (2010). Global soil water balance geospatial database. CGIAR Consort. Spat. Inf. Publ. Online Available CGIAR-CSI GeoPortal Httpwwwcgiar-Csiorg .

Treier, U.A., Broennimann, O., Normand, S., Guisan, A., Schaffner, U., Steinger, T., et al. (2009). Shift in cytotype frequency and niche space in the invasive plant Centaurea maculosa .Ecology , 90, 1366-1377.

Wang, I.J. \& Bradburd, G.S. (2014). Isolation by environment.Mol. Ecol. , 23, 5649-5662.

Wang, I.J., Glor, R.E. \& Losos, J.B. (2013). Quantifying the roles of ecology and geography in spatial genetic divergence. Ecol. Lett. , 16, 175-182.

Warren, D.L., Glor, R.E. \& Turelli, M. (2008). Environmental niche equivalency versus conservatism: quantitative approaches to niche evolution. Evolution , 62, 2868-2883.

Wisz, M.S., Pottier, J., Kissling, W.D., Pellissier, L., Lenoir, J., Damgaard, C.F., et al. (2013). The role of biotic interactions in shaping distributions and realised assemblages of species: implications for species distribution modelling. Biol. Rev. , 88, 15-30.

Wood, T.E., Takebayashi, N., Barker, M.S., Mayrose, I., Greenspoon, P.B. \& Rieseberg, L.H. (2009). The frequency of polyploid speciation in vascular plants. Proc. Natl. Acad. Sci. U. S. A. , 106, 13875-13879.

Zohary, M. (1973). Geobotanical foundations of the Middle East . Gustav Fisher Verlag, Stuttgart, Swets \& Zeitlinger, Amsterdam.

\begin{tabular}{|c|c|c|c|c|c|c|c|c|c|c|}
\hline $\begin{array}{l}\text { Species } \\
\text { (GenomeNłg }\end{array}$ & $\begin{array}{l}\text { Plastid } \\
\text { loci }\end{array}$ & $\begin{array}{l}\text { Plastid } \\
\text { loci }\end{array}$ & $\begin{array}{l}\text { Plastid } \\
\text { loci }\end{array}$ & $\begin{array}{l}\text { Nuclear } \\
\text { loci }\end{array}$ & $\begin{array}{l}\text { Nuclear } \\
\text { loci }\end{array}$ & $\begin{array}{l}\text { Nuclear } \\
\text { loci }\end{array}$ & $\begin{array}{l}\text { Nuclear } \\
\text { loci }\end{array}$ & $\begin{array}{l}\text { Nuclear } \\
\text { loci }\end{array}$ & $\begin{array}{l}\text { Nuclear } \\
\text { loci }\end{array}$ & $\begin{array}{l}\text { Ecogeneticco } \\
\text { structurestru }\end{array}$ \\
\hline & $\mathrm{AR}$ & $h$ & $v$ & & $\mathrm{AR}$ & $h$ & $v$ & Ho & $\begin{array}{l}\mathbf{G}^{\sim} \\
\text { Geo++ }\end{array}$ & $\begin{array}{l}\mathbf{G}^{\sim} \quad \mathbf{G}^{-} \\
\mathbf{G e o}++\mathbf{E}+\end{array}$ \\
\hline
\end{tabular}




\begin{tabular}{|c|c|c|c|c|c|c|c|c|c|c|c|c|}
\hline \multicolumn{2}{|c|}{$\begin{array}{l}\text { Species } \\
\text { (GenomeNg }\end{array}$} & \multirow{2}{*}{$\begin{array}{l}\begin{array}{l}\text { Plastid } \\
\text { loci }\end{array} \\
7.41\end{array}$} & \multirow{2}{*}{$\begin{array}{l}\begin{array}{l}\text { Plastid } \\
\text { loci }\end{array} \\
0.92\end{array}$} & \multirow{2}{*}{$\begin{array}{l}\text { Plastid } \\
\text { loci }\end{array}$} & \multirow[t]{2}{*}{$\begin{array}{l}\text { Nuclear } \\
\text { loci }\end{array}$} & \multirow{2}{*}{$\begin{array}{l}\text { Nuclear } \\
\text { loci }\end{array}$} & \multirow{2}{*}{$\begin{array}{l}\text { Nuclear } \\
\text { loci }\end{array}$} & \multirow{2}{*}{$\begin{array}{l}\begin{array}{l}\text { Nuclear } \\
\text { loci }\end{array} \\
0.005\end{array}$} & \multirow{2}{*}{$\begin{array}{l}\text { Nuclear } \\
\text { loci }\end{array}$} & \multirow{2}{*}{$\begin{array}{l}\begin{array}{l}\text { Nuclear } \\
\text { loci }\end{array} \\
0.450^{* * *} \\
(* * *)\end{array}$} & \multicolumn{2}{|c|}{$\begin{array}{l}\text { Ecogeneticco } \\
\text { structurestru }\end{array}$} \\
\hline $\begin{array}{l}\text { Ae. } \\
\text { cau- } \\
\text { data } \\
\text { (C) }\end{array}$ & 29 & & & & & & & & & & $\begin{array}{l}0.450^{* * *} \\
(* * *)\end{array}$ & \\
\hline $\begin{array}{l}\text { Ae. } \\
\text { co- } \\
\text { mosa } \\
(\mathrm{M})\end{array}$ & 30 & 8.24 & 0.95 & 0.0004 & & 4.77 & 0.50 & 0.007 & 0.06 & $\begin{array}{l}0.264^{* *} \\
(* * *)\end{array}$ & $\begin{array}{l}0.264^{* *} \\
(* * *)\end{array}$ & $\begin{array}{l}0.01 \\
(*)\end{array}$ \\
\hline $\begin{array}{l}\text { Ae. } \\
\text { tauschii } \\
\text { (D) }\end{array}$ & 41 & 5.94 & 0.80 & 0.0003 & & 2.84 & 0.40 & 0.003 & 0.00 & $\begin{array}{l}- \\
0.011 \mathrm{~ns} \\
(*)\end{array}$ & $\begin{array}{l}- \\
0.011 \mathrm{~ns} \\
(*)\end{array}$ & $\begin{array}{l}0 . \\
(*\end{array}$ \\
\hline $\begin{array}{l}\text { Ae. } \\
\text { um- } \\
\text { bel- } \\
\text { lu- } \\
\text { lata } \\
\text { (U) }\end{array}$ & 26 & 3.47 & 0.52 & 0.0001 & & 2.78 & 0.33 & 0.002 & 0.15 & $\begin{array}{l}0.482^{* * *} \\
(* * *)\end{array}$ & $\begin{array}{l}0.482^{* * *} \\
(* * *)\end{array}$ & \\
\hline $\begin{array}{l}\text { Ae. } \\
\text { genic- } \\
u- \\
\text { lata } \\
(U \mathrm{M})\end{array}$ & 81 & 9.02 & 0.98 & 0.0007 & & 5.95 & 0.61 & 0.013 & 0.56 & $\begin{array}{l}0.082 \mathrm{~ns} \\
(* * *)\end{array}$ & $\begin{array}{l}0.082 \mathrm{~ns} \\
(* * *)\end{array}$ & $\begin{array}{l}- \\
0 . \\
(*\end{array}$ \\
\hline $\begin{array}{l}\text { Ae. } \\
\text { tri- } \\
\text { un- } \\
\text { cialis } \\
(U C)\end{array}$ & 79 & 5.29 & 0.79 & 0.0013 & & 3.81 & 0.56 & 0.013 & 0.58 & $\begin{array}{l}0.058 \mathrm{~ns} \\
(* * *)\end{array}$ & $\begin{array}{l}0.058 \mathrm{~ns} \\
(* * *)\end{array}$ & $\begin{array}{l}0 . \\
(*\end{array}$ \\
\hline $\begin{array}{l}\text { Ae. } \\
\text { cylin- } \\
\text { drica } \\
(D C)\end{array}$ & 82 & 2.80 & 0.35 & 0.0002 & & 2.30 & 0.50 & 0.013 & 0.64 & $\begin{array}{l}0.150^{*} \\
(* * *)\end{array}$ & $\begin{array}{l}0.150^{*} \\
(* * *)\end{array}$ & $\begin{array}{l}0.13 \\
(*)\end{array}$ \\
\hline $\begin{array}{l}\text { Ae. } \\
\text { crassa } \\
\text { (MD) }\end{array}$ & 10 & 3.00 & 0.38 & 0.0000 & & 3.30 & 0.63 & 0.017 & 0.66 & $\begin{array}{l}0.261 \mathrm{~ns} \\
(* * *)\end{array}$ & $\begin{array}{l}0.261 \mathrm{~ns} \\
(* * *)\end{array}$ & $\begin{array}{l}- \\
0 . \\
(\mathrm{n}\end{array}$ \\
\hline
\end{tabular}

Table 1: Genetic diversity and eco-genetic structure within diploid and allopolyploid Aegilops species. For each species, Ng individuals were genotyped at plastid and nuclear loci to estimate allelic richness (AR), observed heterozygosity (Ho), genetic diversity corrected for sample size $(h)$ and with ordered alleles $(v)$. The eco-genetic structure was assessed through (partial) Mantel tests associating genetic (G), climatic (E) and geographical (Geo) distances among individuals within each species and decomposed into significant isolation-by-distance $\left(\mathrm{G}^{\sim} \mathrm{Geo}\right)$ and isolation-by-environment $\left(\mathrm{G}^{\sim} \mathrm{E} \mid \mathrm{Geo}\right)$. Range filling represents the fraction of suitable areas based on the climatic niche that is occupied by the species within the study area.

+ Genome composition of each species according to van Slageren (1994), with maternal progenitor underlined ++ Associations tested through 20000 permutations with p-values as $* * *<0.001,{ }^{* *}<0.01,{ }^{*}<0.05$ and ns for non-significant and confirmed through Redundancy Analysis whose significance is indicated in between brackets. 
Fig. 1: Genetic and ecological additivity of diploid progenitors as a null hypothesis to analyze micro-evolution of allopolyploid species. Multilocus genotypes are shown for diploid (stars) and allopolyploid (diamonds) individuals over an adaptive landscape presenting fitness as grey isolines. On the left, ecological niches of diploid species are overlaid as shaded areas delimiting populations existing across the environmental space. Allopolyploids combine divergent suites of genes (shown as - or + between brackets) from diploid species (AoAo and BoBo) and are therefore expected to present genetic additivity (AeAeBeBe). Fixed heterozygosity for genes underlying the ecological niche of each diploid progenitor is expected to provide positive population growth over combined ranges of environmental conditions tolerated by progenitor species. As a null hypothesis, allopolyploid speciation therefore produces new species presenting the ecological additivity of their progenitors, with an observed niche (AoAoBoBo) similar to the expected niche (i.e. $\mathrm{AeAeBeBe}$ ). On the right, main scenarios of possible ecological niche evolution during the establishment of allopolyploids are presented. Ecological niches of allopolyploids can retain the additivity of niches from their diploid progenitors. Significant deviation from such expected additivity is however characteristic of niche shifts in allopolyploids. Niche contraction occurs when allopolyploids do not outcompete diploid progenitors and occupy a subset of their niches. In contrast, niche expansion beyond the combined range of conditions tolerated by diploid progenitors is coherent with evolutionary novelties commonly postulated to promote allopolyploid establishment in face of their competition. Analyses relying on such expected additivity of diploids adequately test for multivariate niche conservatism vs shift in allopolyploids and, circumventing ambiguous pairwise comparisons with divergent progenitors, transcend diagnostics based on niche centroids (crosses) and/or niche breadths (braces along univariate ecological gradients). Verifiable conclusions are accordingly reached regarding niche conservatism vs novelty in allopolyploids responding to variable competition from diploids in space and time.

Figure 2: Evolution of Aegilops allopolyploid species following the merging of diploid progenitors. A. Phylogenetic relationships within and among four diploids species Ae. tauschii(DD), Ae. caudata (CC), Ae. umbellulata (UU) and Ae. comosa (MM), and four derived allopolyploid species Ae. cylindrica (DDCC), Ae. triuncialis (UUCC), Ae. geniculata(UUMM) and Ae. crassa (DDMM with tetraploids, 4x, and hexaploids, $6 \mathrm{x}$ ). Origins of allopolyploid species were inferred through independent multi-labelled trees based on 30 nuclear loci anchored in the phylogeny of diploid wheats (Fig. S3). Divergence from maternal (plain line) and paternal (dashed line) progenitors are reported with corresponding dates. Tips present accessions from genetic clusters identified with the same loci, labelled according to Fig. 3. Maternal progenitor species of allopolyploids, identified using plastid loci, are underlined (Fig. S2).B. Nuclear genomic composition of the four allopolyploid species estimated as the proportion of conserved (blue), derived (orange) and lost (grey) haplotypes across loci as compared to those segregating within their diploid progenitors (Table S2).

Figure 3: Spatial genetic structure within four diploid and four derived allopolyploid Aegilops species. Genotyped accessions (circles) are assigned to genetic clusters inferred by STRUCTURE within each species, colored according to the stacked barplots underneath and approximately delimited across distribution ranges. Contact zones between genetic clusters showing admixed accessions are indicated by red stripes. Labels are otherwise as in Fig. 2.

Figure 4: Climatic niche dynamics of allopolyploid Aegilops species. Climatic niche of each Aegilops species in the common climatic space ( $100 \%$ and $50 \%$ indicated as solid and dashed contour lines) along the first two principal components based on 20 bioclimatic factors shown in the central correlation circle (abbreviations in Fig. S6). The observed niche of each polyploid species is compared to the expected additivity of climatic niches modeled from both its diploid progenitors (blue + green). The fraction of the expected niche effectively occupied by the allopolyploid species characterizes niche stability (blue), whereas the unoccupied fraction is referred to as niche contraction (green). Climatic space occupied by the allopolyploids while not predicted by the additivity of diploid progenitors indicates niche expansion (red). For each allopolyploid, niche centroids of diploid progenitors are indicated as orange stars labelled with their genome composition. Labels are otherwise as in Fig. 2. 

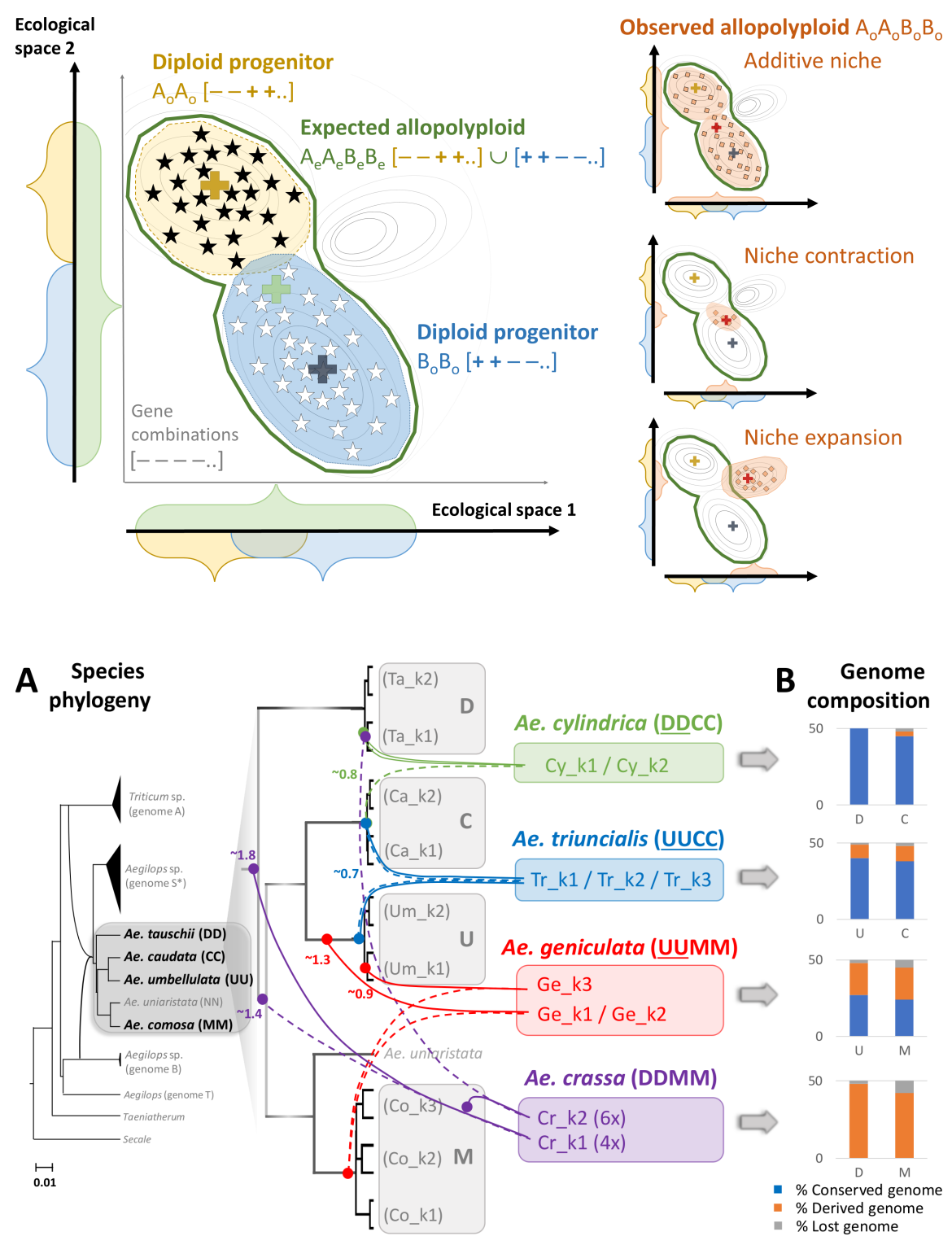

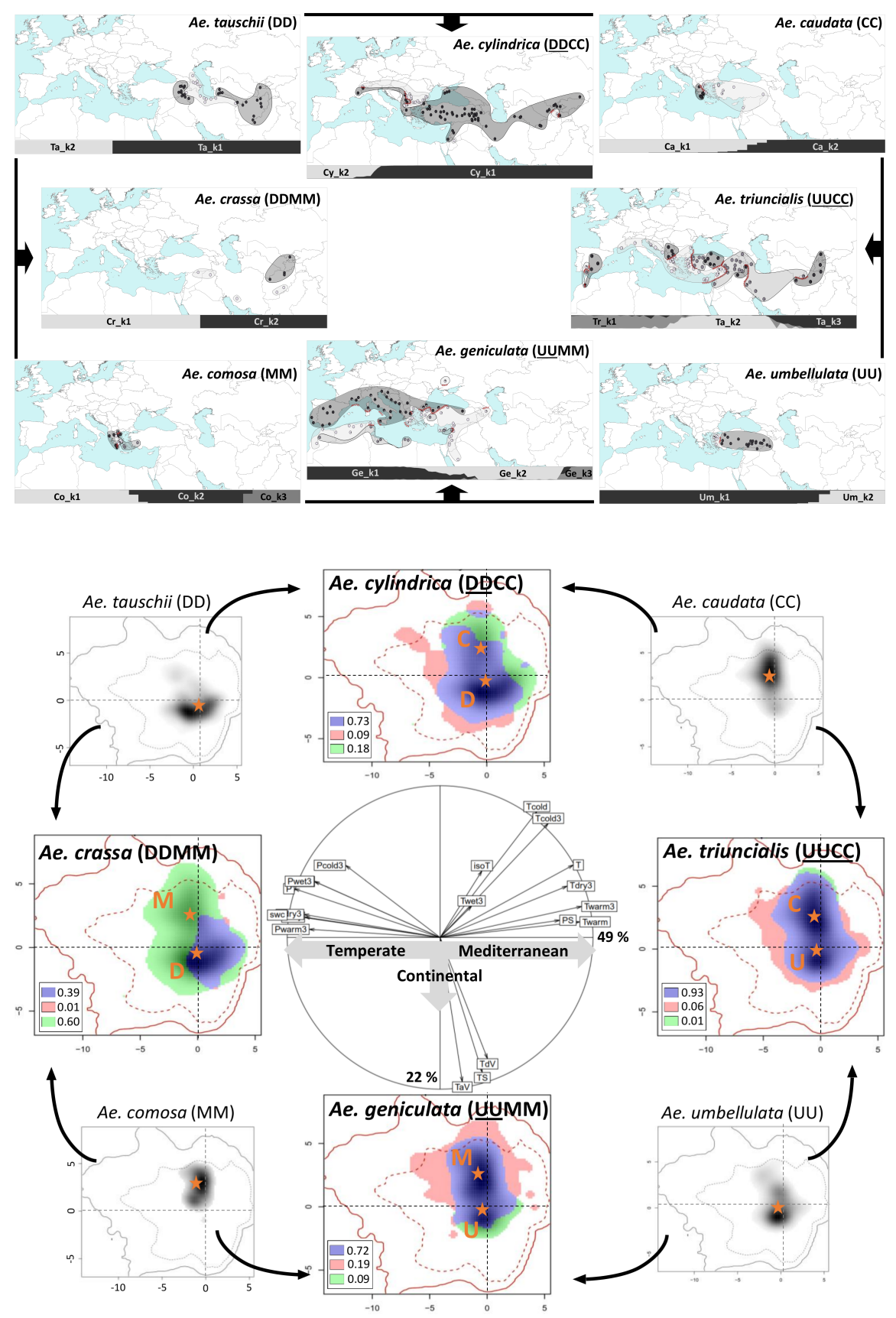